Check for updates

Cite this: Analyst, 2020, 145, 7071

\title{
Correction: Investigation of heart lipid changes in acute $\beta$-AR activation-induced sudden cardiac death by time-of-flight secondary ion mass spectrometry
}

\author{
Jia-Qian Lou, $\dagger^{\mathrm{a}}$ Yue Cao, $\uparrow^{\mathrm{a}}$ You-Jia Yu, ${ }^{\mathrm{a}} \mathrm{Li} \mathrm{Hu},{ }^{a}$ Zheng-Sheng Mao, ${ }^{\mathrm{a}}$ Ping Huang, ${ }^{\mathrm{b}}$ \\ Xin Hua*c and Feng Chen*a,d
}

DOI: 10.1039/d0an90092c

Correction for 'Investigation of heart lipid changes in acute $\beta$-AR activation-induced sudden cardiac death by time-of-flight secondary ion mass spectrometry' by Jia-Qian Lou, et al., Analyst, 2020, DOI: 10.1039/

rsc.li/analyst d0an00768d

The authors regret that there were errors in some of the units provided in section 2.6 of the original article. The corrected section 2.6 is shown below:

ToF-SIMS analysis was performed using a ToF-SIMS V instrument (ION-TOF GmbH, Munster, Germany) with a $30 \mathrm{keV} \mathrm{Bi}_{3}{ }^{+}$ primary ion gun, which hits targets at an angle of $45^{\circ}$. The area of analysis for mass spectra and chemical mapping was $500 \times$ $500 \mu \mathrm{m}^{2}$, the primary ion dose density (PIDD) was $4.09 \times 10^{11} \mathrm{~cm}^{-2}$ and a supplementary larger field of adaptive size for each tissue was used to observe regional differences. NC scan area: $3 \times 3.5 \mathrm{~mm}^{2}$, SCD scan area: $7 \times 5 \mathrm{~mm}^{2}$, PIDD for positive mode: $7.99 \times 10^{10} \mathrm{~cm}^{-2}$. The scan area of human samples is $3 \times 3 \mathrm{~mm}^{2}$ and PIDD is $1.25 \times 10^{10} \mathrm{~cm}^{-2}$. The target current was $\sim 1.0 \mathrm{pA}$. The mass resolution $(m / \Delta m)$ was 4000 for $\mathrm{C}_{2} \mathrm{H}_{5}{ }^{+}$. Positive ion mass spectra were calibrated using the $\mathrm{C}^{+}, \mathrm{CH}^{+}, \mathrm{CH}_{2}{ }^{+}, \mathrm{CH}_{3}{ }^{+}, \mathrm{C}_{2} \mathrm{H}_{3}{ }^{+}$, $\mathrm{C}_{2} \mathrm{H}_{5}{ }^{+}, \mathrm{C}_{3} \mathrm{H}_{5}{ }^{+}$and $\mathrm{C}_{5} \mathrm{H}_{15} \mathrm{NPO}_{4}{ }^{+}$peaks. Three mass spectra of regions of interest for each section in positive polarities were collected. ToF-SIMS results were obtained and analyzed using IONTOF instrument software (SurfaceLab 7.0).

In addition, the authors regret the omission of citations to Fig. S1, Fig. 3C and Table S2 in the original article. The following sections of text in the manuscript should be adjusted as detailed below:

The sentence on page 5892 beginning "The results indicated that DAG..." should be correctly given as "The results indicated that DAG decreased, while LysoPC and PC increased in SCD mice (Fig. S1).”

The sentence on page 5892 beginning "In Fig. 3B, the loading plots show..." should be correctly given as "In Fig. 3B-C, the loading plots show that the major contributions from DAGs $(\mathrm{m} / \mathrm{z} 519.33,521.31,523.34,547.47,549.41,551.49,573.36,575.37$, $577.38,599.51,601.56,603.55$, and 605.49) were utilized to separate the NC myocardium from the SCD myocardium, while cholesterol ( $\mathrm{m} / \mathrm{z} 369.31$ and 385.31$)$ had a minor contribution."

The sentence on page 5894 beginning "These human results shared similarities..." should be correctly given as "These human results shared similarities to those obtained from mice (Table S2), except that Vita-E $(\mathrm{m} / \mathrm{z} 430.37)$ was detected in both H-NC and H-CASE and increased in H-CASE."

The Royal Society of Chemistry apologises for these errors and any consequent inconvenience to authors and readers.

\footnotetext{
${ }^{a}$ Department of Forensic Medicine, Nanjing Medical University, Nanjing, China. E-mail: fchen@njmu.edu.cn

${ }^{b}$ Shanghai Key Laboratory of Forensic Medicine, Shanghai Forensic Service Platform, Academy of Forensic Science, Shanghai, 200063, China

${ }^{c}$ Department of Chemistry, East China University of Science and Technology, 130 Meilong Road, Shanghai200237, China. E-mail: xinhua@ecust.edu.cn

${ }^{d}$ Key Laboratory of Targeted Intervention of Cardiovascular Disease, Collaborative Innovation Center for Cardio-vascular Disease Translational Medicine, Nanjing Medical

University, Nanjing, Jiangsu, 211166, PR China

$\dagger$ These authors contributed equally to this work.
} 